\title{
Cloning and Expression Analysis of MeTCP4 Transcription Factor from Cassava and Construction of Plant Expression Vector
}

Lei Ning ${ }^{1,2}$, Li Shuxia $^{1}$, Peng Ming 1

1 Institute of Tropical Bioscience and Biotechnology, Chinese Academy of Agriculture Sciences, Haikou, 571101, China

2 College of Tropical Agriculture and Forestry, Hainan University, Haikou, 570228, China

$\varnothing$ Corresponding author email: pengming@itbb.org.cn

Molecular Plant Breeding, 2019, Vol.10, No.5 doi: $10.5376 / \mathrm{mpb} .2019 .10 .0005$

Received: 21 Jan., 2019

Accepted: 20 Feb., 2019

Published: 30 Jan., 2019

Copyright ㅇ 2019 Lei et al., This article was first published in Molecular Plant Breeding (2018, 05: 1517-1523) in Chinese, and here was authorized to translate and publish the paper in English under the terms of Creative Commons Attribution License, which permits unrestricted use, distribution, and reproduction in any medium, provided the original work is properly cited.

Preferred citation for this article:

Lei N., Li S.X., and Peng M., 2019, Cloning and expression analysis of MeTCP4 transcription factor from cassava and construction of plant expression vector, Molecular Plant Breeding, 10(5): 34-42 (doi: $\underline{10.5376 / \mathrm{mpb} .2019 .10 .0005)}$

\begin{abstract}
The TCP transcription factor family genes are involved in multiple regulation of plant growth and environmental stress. However, these family genes are rarely studied in cassava (Manihot esculenta). There are 36 TCP genes in cassava. Phylogenetic tree analysis indicates that MeTCP can be divided into 8 subgroups. MeTCP4 is one of 36 genes in cassava TCP family, which can be transcribed by miR319 and sheared. MeTCP4 contained an open reading frame (ORF) of 1269 bp, which encoded 422 amino acids with a predicted molecular weight of $45.75 \mathrm{kD}$ and theoretical PI of 6.17. The full-length cDNA sequence of MeTCP4 was cloned from the cassava genome. The results of quantitative RT-PCR showed that the expression of MeTCP4 can be found in all the tested tissues, with the lowest in roots, second in stems and the highest in leaves. MeTCP4 gene was repressed by drought and low temperature stress. Construction of high and low plant expression vector would provide basis for further study of the function of the gene.
\end{abstract}

Keywords Cassava (Manihot esculenta); Gene clone; MeTCP4 transcription factor; qRT-PCR; Expression vector construction

\section{Background}

Cassava (Manihot esculenta) is a perennial shrub, one of the six major cash crops in the world. It is widely cultivated in tropical and subtropical regions of Asia, Africa and Latin America, and eaten by more than 5 billion people (El-Sharkawy, 2004). The research pointed out that cassava (Manihot esculenta) has relative advantages in biological productivity compared with the main tropical food and energy crops. Compared with other crops, this crop has higher yield potential under good conditions and shows superiority under sub-optimal conditions, thus providing the possibility of increasing total agricultural yield by using marginal land (Cock, 1982). The crop will play a greater role in tropical and subtropical agro-ecosystems under global warming conditions. Cassava (Manihot esculenta) has a wide range of rooting systems, and its stomata are highly sensitive to atmospheric humidity and soil water shortage. These characteristics are the physiological basis of drought tolerance of cassava (El-Sharkawy, 1993). In addition, cassava (Manihot esculenta) has the characteristics of high light efficiency, high starch yield, barren tolerance and stress resistance, which fully illustrates that cassava (Manihot esculenta) has high research value. So, it is necessary to explore the characteristics of cassava from the genetic level.

In natural growth environment, plants may be affected by environmental stress factors at any time. Environmental stress can be divided into two types: biotic stress and abiotic stress (e.g. low temperature, drought, high salinity, etc.). Abiotic stress has the most common impact on plant growth. Plants must respond to and adapt to abiotic stress in order to survive under various environmental conditions (Urano et al., 2010). Among abiotic stresses, drought and low temperature are the most common stress factors. Low temperature and drought can cause water stress (decrease of swelling pressure, disorder of normal metabolism, decrease of water potential of plant tissues, etc.), and their effects on plant growth are also the most common, resulting in low temperature and drought becoming the most serious abiotic stress factors (Mahajan and Tuteja, 2005). At present, many genes related to drought and salt tolerance in plants have been cloned and analyzed. At the same time, these genes can be 
transferred to plants for heterologous expression through transgenic technology, which can significantly improve the drought and salt tolerance level of transgenic plants (Chen and He, 2010). From the perspective of gene function, the study of plant drought resistance and cold tolerance will help people to understand the mechanism of plant adaptation to abiotic stress deeply, so as to understand and improve plants from a genetic perspective.

TCP family genes were first named after four similar but unrelated protein coding genes: maize $T B 1$, goldfish grass $C Y C$, rice $P C F 1$ and $P C F 2$ genes (Cubas et al., 1999). The proteins encoded by TCP family genes have distinct TCP domains. TCP domains are an atypical Basic-Helix-Loop (bHLH) structure composed of 56 amino acids, which can bind to DNA and interact with proteins (Luo et al., 1996; Doebley et al., 1997; Kosugi and Ohashi, 1997). Twenty-four TCP genes have been identified by whole-genome search in Arabidopsis thaliana (Riechmann et al., 2000). The TCP family genes of cassava, a tropical and subtropical crop, have not been reported yet, so the research of cassava (Manihot esculenta) TCP family related genes is imminent. Cassava (Manihot esculenta), as a tropical crop, is highly barren-tolerant and drought-tolerant, which endows it with excellent gene function. From the perspective of TCP transcription factors, this study aims to reveal the molecular mechanism of cassava to respond to drought and low temperature stress.

We found that cassava contains $36 \mathrm{TCP}$ family genes. In order to verify some functions of the family genes in cassava, MeTCP4 gene was selected as the research object. MeTCP4 gene was cloned and its expression pattern was analyzed, and its high/low expression vector was constructed to lay a foundation for further research on the function and mechanism of MeTCP4 gene and provide support for cassava molecular breeding.

\section{Results and Analysis}

\subsection{Family bioinformatics analysis}

In order to study the evolutionary relationship of MeTCP family members in cassava, the phylogenetic tree of 36 TCP family genes were constructed by Neighbor-joining of MEGA7.0 software. The members of cassava TCP gene family could be divided into eight groups (I-VII). There are 4 genes in group I, 4 genes in group II, 3 genes in group III, 5 genes in group IV, 6 genes in group V, 5 genes in group VI, 5 genes in group VII and 4 genes in group VIII (Figure 1).

\subsection{Total RNA extraction and cDNA synthesis}

Cassava (Manihot esculenta) total RNA was extracted by polysaccharide polyphenol plant total RNA extraction kit (Tiangen \# DP140916). RNA content was determined by NanoDrop2000 (Thermo scientific). The integrity of the extracted RNA was further detected by $1 \%$ agarose gel electrophoresis, and $18 \mathrm{~S}$ and $28 \mathrm{~S}$ rRNA were clearly visible (Figure 2). The results showed that the concentration and purity of total RNA extracted from cassava were good and could be used in subsequent experiments. Cassava total RNA was used as template to synthesize cDNA using reverse transcription kit.

\subsection{Characteristic analysis of gene cloning, sequence and encoding protein}

Cassava (Manihot esculenta) MeTCP4 gene sequence was obtained from cassava genome database (https://phytozome.jgi.doe.gov/pz/portal.html\#). ORF sequence analysis on MeTCP4 gene was performed using geneious software (http://www.geneious.com/). The results showed that it had a complete ORF sequence and the full length of CDS was $1269 \mathrm{bp}$. Specific PCR primers were designed, and cassava cDNA was used as template. After specific PCR amplification, MeTCP4 full-length sequence was finally obtained. Then $1 \%$ agarose gel electrophoresis was used for detection (Figure 3) and the product was recovered. Finally, the vector was transformed into Escherichia coli DH5 $\alpha$ competent cells, with overnight cultivation, resistance screening and bacterial liquid detection. Sequencing results were consistent with genomic data. Cassava (Manihot esculenta) MeTCP4 gene encoded 422 amino acids. By expasy software (http://web.expasy.org/protparam/) analysis (Zhangfan et al., 2017), the relative molecular weight of the protein encoded by cassava TCP4 gene was $45.75 \mathrm{kD}$, with the theoretical isoelectric point of 6.17 and the Gravy value of -0.636 . 


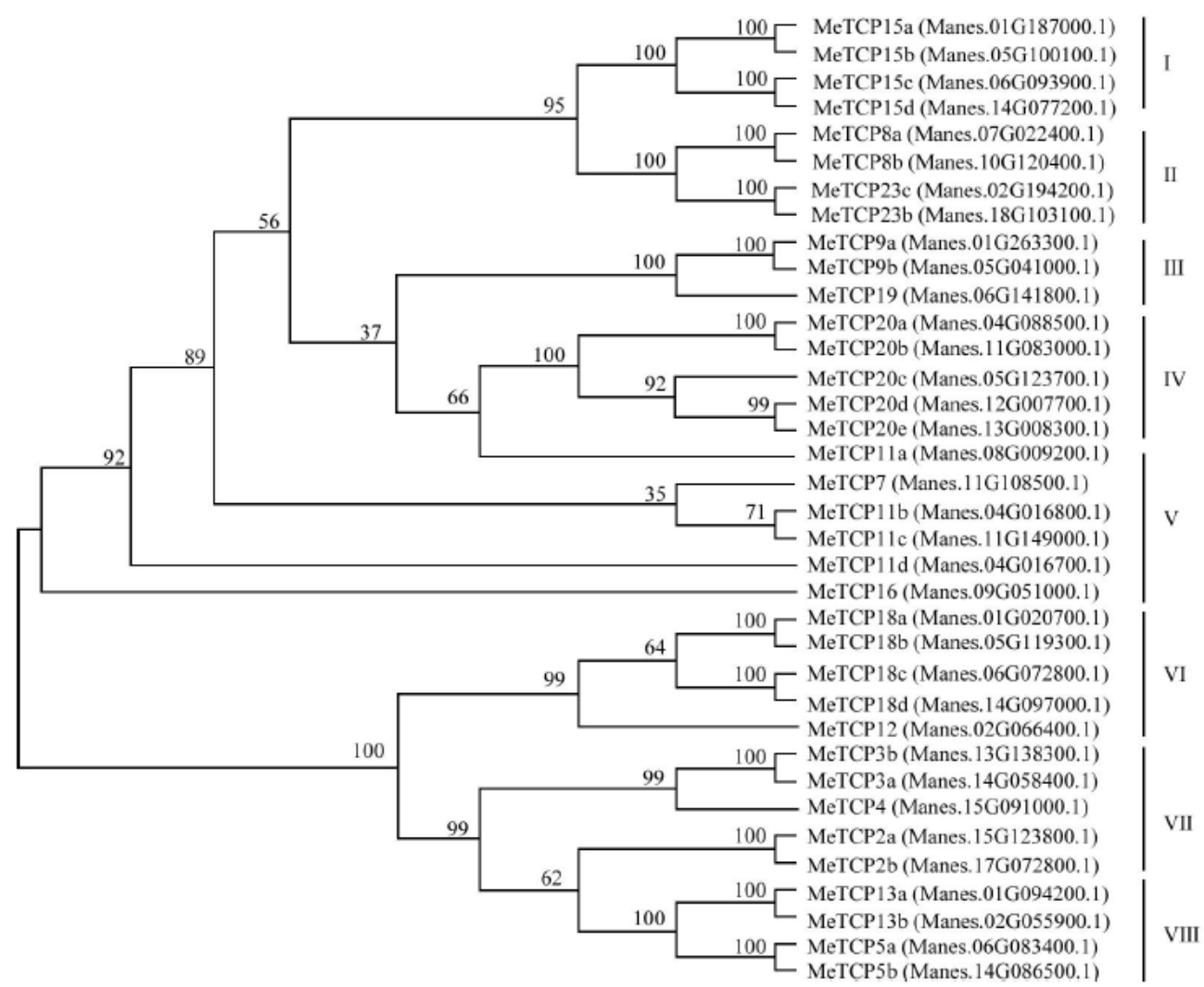

Figure 1 Phylogenetic tree of MeTCP family members in cassava

Note: I: MeTCP15a (Manes.01G187000.1), MeTCP15b (Manes.05G100100.1), MeTCP15c (Manes.06G093900.1), MeTCP15d (Manes.14G077200.1); II: MeTCP8a (Manes.07G022400.1), MeTCP8b (Manes.10G120400.1), MeTCP23a (Manes.02G194200.1), MeTCP23b (Manes.18G103100.1); III: MeTCP9a (Manes.01G263300.1), MeTCP9b (Manes.05G041000.1), MeTCP19 (Manes.06G141800.1); IV: $\quad$ MeTCP20a (Manes.04G088500.1), $\quad$ MeTCP20b (Manes.11G083000.1), MeTCP20c (Manes.05G123700.1), $\quad$ MeTCP20d $\quad$ (Manes.12G007700.1), $\quad$ MeTCP20e $\quad$ (Manes.13G008300.1); $\quad$ V: $\quad$ MeTCP11a (Manes.08G009200.1), MeTCP7 (Manes.11G108500.1), MeTCP11b (Manes.04G016800.1), MeTCP11c (Manes.11G149000.1), MeTCP11d (Manes.04G016700.1), MeTCP16 (Manes.09G051000.1); VI: MeTCP18a (Manes.01G020700.1), MeTCP18b (Manes.05G119300.1), MeTCP18c (Manes.06G072800.1), MeTCP18d (Manes.14G097000.1), MeTCP12 (Manes.02G066400.1); VII: MeTCP3b (Manes.13G138300.1), MeTCP3a (Manes.14G058400.1), MeTCP4 (Manes.15G091000.1), MeTCP2a (Manes.15G123800.1), $\quad$ MeTCP2b (Manes.17G072800.1); $\quad$ VIII: $\quad$ MeTCP13a $\quad$ (Manes.01G094200.1), MeTCP13b (Manes.02G055900.1), MeTCP5a (Manes.06G083400.1), MeTCP5b (Manes.14G086500.1)

RNA

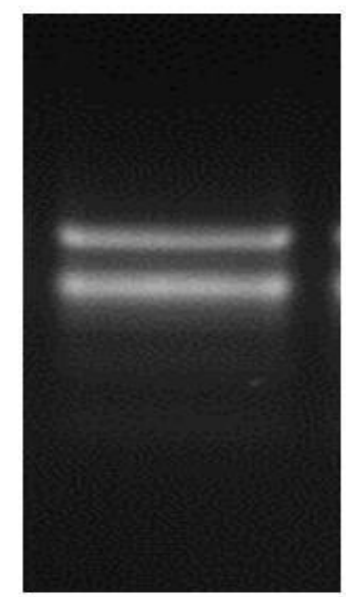

Figure 2 The total RNA extracted from cassava 


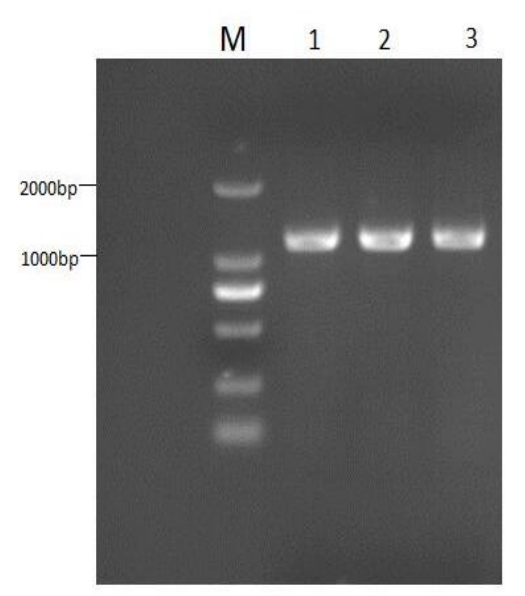

Figure 3 The PCR amplification product of MeTCP4 gene

Note: M: D2000 DNA Marker; 1,2,3: MeTCP4

\subsection{Analysis of genomic tissues distribution expression and expression patterns under adversity stress}

The total RNA of roots, stems and leaves of cassava (Manihot esculenta) seedlings were extracted respectively. The integrity of RNA was detected by gel electrophoresis. Two distinct bands were observed under UV after electrophoresis, named $28 \mathrm{~S}$ and $18 \mathrm{~S}$, respectively (Figure 4). Total RNA could be used for subsequent tissue-specific expression analysis. qRT-PCR analysis showed that cassava (Manihot esculenta) MeTCP4 gene had obvious tissue expression specificity, with the lowest expression in root, the second lowest in stem and the highest expression in leaf (Figure 5).

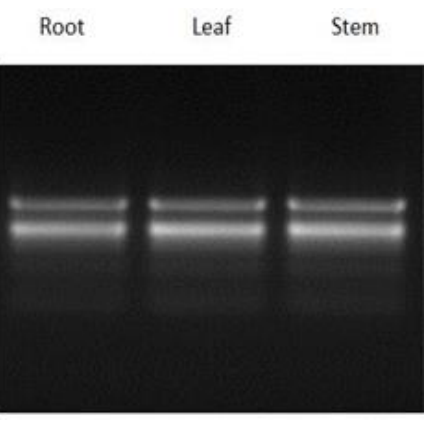

Figure 4 The total RNA in root, stem and leaves of cassava

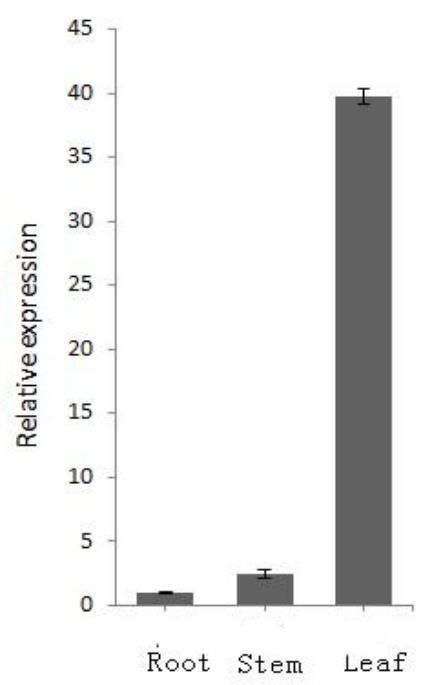

Figure 5 Expression of cassava MeTCP4 gene in root, stems and leaves 
To verify whether the expression of MeTCP4 gene in cassava was affected by low temperature and drought stress, the expressions of MeTCP4 gene in cassava were studied under drought and low temperature stress, respectively. qRT-PCR analysis after low temperature treatment showed that (Figure 6): Cassava (Manihot esculenta) MeTCP4 gene responded to low temperature in roots, stems and leaves, and MeTCP4 gene had different expression patterns in roots, stems and leaves. Compared with the control, the expression level reached the peak in stem and the lowest in leaf after 6 hours of low temperature treatment. In general, the MeTCP4 gene in roots and leaves showed a downward trend after 6 hours of treatment. It was noteworthy that the expression of MeTCP4 gene in leaves decreased gradually from 1 hour after treatment, while the expression of MeTCP4 gene in stems increased first and then decreased. qRT-PCR analysis after drought treatment showed that (Figure 5): The expression of MeTCP4 gene reached the peak in leaves and roots after 6 hours of PEG treatment, and the lowest expression was found in stem after 3 hours of PEG treatment. In general, the expression of MeTCP4 gene in roots, stems and leaves treated with PEG showed a downward trend.

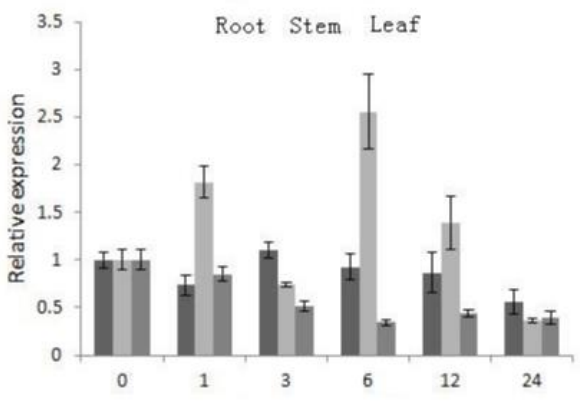

Time of $4^{\circ} \mathrm{C}$ Treatment (h)

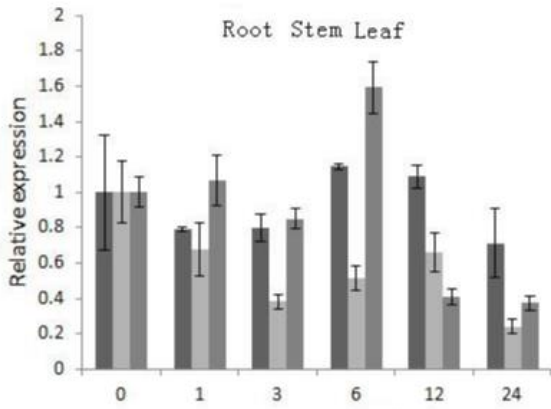

Time of PEG Treatment (h)

Figure 6 Expression patterns of MeTCP4 treated with abiotic stresses

\subsection{Construction of plant expression vector}

BamH1 and Sal 1 were used to digest rMeTCP4 and MeTCP4-SRDX which were resistant to the cleavage of miR319 and were linked into pCAMBIA1301 vector to construct plant high expression vector pCAMBIA1301-35S:: rMeTCP4 and low expression vector pCAMBIA1301-35S:: MeTCP4SRDX plasmid. Transferred into E. coli DH5a, the positive recombinant plasmid bacteria solution was detected by PCR (Figure 7) and sequenced. The electrophoresis of positive bacterium solution PCR results showed that the size of fragment was about $1269 \mathrm{bp}$, which was consistent with the sequencing results. The positive strains with correct sequencing were cultured overnight and the plasmids were extracted and stored at $-20^{\circ} \mathrm{C}$ for later use.

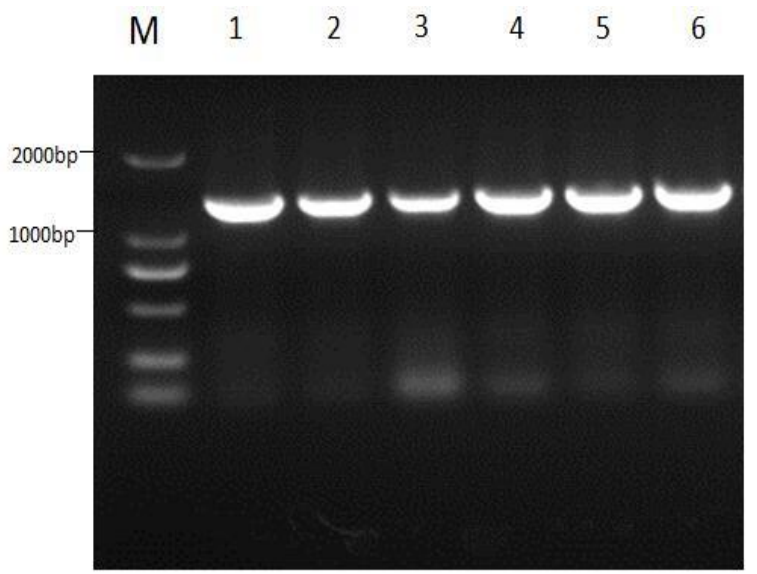

Figure 7 PCR verification of plant expression vector pCAMBIA1301- 35S::rMeTCP4/ pCAMBIA1301- 35S::MeTCP4SRDX bacterium solution

Note: M: D2000 DNA Marker; 1,2,3: pCAMBIA1301- 35S::rMeTCP4; 4,5,6: pCAMBIA1301- 35S::MeTCP4SRDX 


\section{Discussion}

Drought and low temperature stress seriously affect plant growth and development, and limit crop yield. TCP family genes are plant specific transcription factors, which play an important role in plant growth and development and abiotic stress response. At present, there are many reports about TCP family genes in many plants. As a tropical and subtropical crop, there are no reports about TCP family genes in cassava (Manihot esculenta). The molecular mechanism of cassava in response to drought and low temperature stress is not clear. This study would help us to understand TCP family genes in cassava (Manihot esculenta) development and abiotic stress response.

There are 36 genes in cassava TCP family. The phylogenetic tree analysis showed that the cassava (Manihot esculenta) TCP family genes could be clustered into eight subfamilies: group I had four genes: MeTCP15a, MeTCP15b, MeTCP15c, MeTCP15d; group II had four genes: MeTCP8a, MeTCP8b, MeTCP23a, MeTCP23b; group III had three genes: MeTCP9a, MeTCP9b, MeTCP19; group IV had five genes: MeTCP20a, MeTCP2Ob, MeTCP20c, MeTCP2Od, MeTCP20e; group V had six genes: MeTCP11a, MeTCP7, MeTCP11b, MeTCP11c, MeTCP11d, MeTCP16; group VI had five genes: MeTCP18a, MeTCP18b, MeTCP18c, MeTCP18d, MeTCP12; group VII had five genes: МeTCP3b, МeTCP $3 a, M e T C P 4, M e T C P 2 a, M e T C P 2 b$; group VIII had four genes: MeTCP13a, MeTCP13b, MeTCP5a, MeTCP5b. There were significant differences among the subgroups of TCP family genes in cassava. The members of IV, V, VI and VII subgroups had 5, 6, 5 and 5 genes, respectively, while the least subgroup III had only 3 genes. The differences among members of different subgroups of the TCP family phylogenetic tree proved that cassava (Manihot esculenta) had undergone gene functional differentiation and specific generation reproduction in the long evolutionary process.

In order to understand the gene expression pattern of cassava (Manihot esculenta) TCP family, MeTCP4 (Manes.15G091000.1) gene was selected as the research object. MeTCP4 gene was cloned for the first time and its tissue-specific expression was analyzed. The results of qRT-PCR showed that MeTCP4 was expressed in roots, stems and leaves, with the lowest expression in roots, followed by stems and the highest expression in leaves. Plant leaves had a series of physiological functions such as photosynthesis and respiration. When plants were under abiotic stresses such as low temperature and drought, water loss of plant leaves would be more serious. In order to adapt to adverse environment, plant leaf organs were particularly important. Therefore, MeTCP4 gene might have potential functions in abiotic stresses such as drought and low temperature.

Under low temperature and drought stress, qRT-PCR results showed that the expression of MeTCP4 gene in cassava (Manihot esculenta) stems increased first and then decreased after low temperature and drought treatment, and the overall trend was downward. The expression level of MeTCP4 gene reached the lowest in leaves of cassava (Manihot esculenta) treated with low temperature at $6 \mathrm{~h}$, while MeTCP4 gene reached the lowest level in leaves treated with drought at $12 \mathrm{~h}$. One-month old cassava (Manihot esculenta) tissue culture seedlings were selected for low temperature treatment. The growth environment of cassava (Manihot esculenta) tissue culture seedlings was sealed in the culture bottle, and the relative humidity of its growth environment was high. Plants were subjected to low temperature and drought stress, which could both cause water stress. The water loss rate of cassava seedlings during low temperature treatment was not exactly the same with that of natural environment, so the expression level of MeTCP4 gene was not significantly different from that of the control group, but the overall trend was consistent. The study pointed out that AtTCP4 gene was the target gene of miR319 in Arabidopsis thaliana, miR319 negatively regulated AtTCP4 gene (Riechmann et al., 2000; Schommer et al., 2008), and cassava MeTCP4 gene was also the target gene of miR319, which was negatively regulated by miR319. Studies had shown that over-expression of miR319 transgenic Agrostiic stolonifera L. could improve its salt and drought tolerance (Zhou et al., 2013). When rice was stressed by low temperature, overexpression miR319 gene could significantly improve the tolerance of rice to low temperature. It was also demonstrated that low expression of negative regulatory target genes OSPCF6 and OSTCP21, OsPCF6 and OSTCP21 by miR319 over-expressing enhanced the tolerance of rice to low temperature (Wang et al., 2014). Our qRT-PCR results also showed that the expression of MeTCP4 gene was overall down-regulated trend after low temperature and drought treatment to 
cassava (Manihot esculenta). In conclusion, cassava (Manihot esculenta) MeTCP4 gene was very likely to mediate cassava response to drought and low temperature stress as an important negative regulator.

SRDX is a small peptide with inhibitory domain composed of 12 amino acids. Even in the presence of redundant genes, it can effectively inhibit the function of target genes and their downstream target genes of redundant genes, and produce phenotypes similar to deleted mutants (Hiratsu et al., 2003). This method has been widely used in the study of transcription factor gene function (Koyama et al., 2007). The modified CDS sequence was named MeTCP4SRDX, and the low expression vector pCAMBIA1301-35S::MeTCP4SRDX was constructed by using the $35 \mathrm{~S}$ constitutive promoter of tobacco mosaic virus to drive MeTCP4SRDX. MeTCP4 could be cut by miR319. If only the original sequence of MeTCP4 gene was overexpressed, the effect of overexpression might not be achieved. Therefore, by introducing a point mutation at the binding site of MeTCP4 and miR156, we antagonized miR319 to shear it without changing its coding amino acid sequence. The modified CDS was named rMeTCP4. We constructed a high expression vector pCAMBIA1301-35S:: rMeTCP4 by using the promoter of tobacco mosaic virus $35 \mathrm{~S}$ to drive $r M e T C P 4$. In order to further verify the function of MeTCP4 gene, the next experiment would use 35S::MeTCP4-SRDX and 35S:: rMeTCP4 to study on transgenic cassava (Manihot esculenta) with $M e T C P 4$ gene and further understand its role in abiotic stress.

In this study, $36 \mathrm{MeTCP}$ genes in cassava were analyzed by bioinformatics. To preliminarily understand the response of MeTCP family genes to abiotic stress, MeTCP4 was selected as the research object and elaborated at the level of gene and tissue expression analysis. At present, the effect of cassava (Manihot esculenta) MeTCP family and MeTCP4 gene on abiotic stresses such as low temperature and drought was only speculated. This study laid a foundation for the next step of MeTCP4 transgenic cassava (Manihot esculenta), and had important guiding significance for further revealing the function of MeTCP4 gene and MeTCP family gene in cassava (Manihot esculenta).

\section{Materials and Methods}

\subsection{Materials}

Cassava variety C3 was from the Laboratory of Functional Genome and Bioengineering, Institute of Biotechnology, Chinese Academy of Tropical Agricultural Sciences. Escherichia coli DH5 $\alpha$ was the reserved strain in our laboratory. Polysaccharide Polyphenol Plant Total RNA Extraction Kit (Directory No: DP441) and Fast Quant RT Kit (Directory No: KR106) synthesized by the first chain of cDNA were purchased from Beijing Tiangen Biochemical Technology Co., Ltd. DNA Purification and Recovery Kit (Catalogue No. 209) was purchased from Shanghai Biotechnology Bioengineering Co., Ltd. Prime STAIR Max DNA polymerase Kit for DNA Amplification (Cat\#RO45A) was purchased from TaKaRa Company. Real-time fluorescent quantitative PCR kit SYBR Premix Ex Taq II (Cat\#RR820A) was purchased from Clontech Company. Primer sequencing and synthesis were completed by Shanghai Biotechnology and Bioengineering Co., Ltd.

\subsection{Plant processing method}

Tissue culture seedlings of one month old cassava variety $\mathrm{C} 3$ with the same growth trend were selected and treated at low temperature and drought for $0,1 \mathrm{~h}, 3 \mathrm{~h}, 6 \mathrm{~h}, 12 \mathrm{~h}$, and $24 \mathrm{~h}$. Low temperature treatment was carried out in light incubator (incubation conditions: temperature $4^{\circ} \mathrm{C}$, relative humidity $60 \%$, light $16 \mathrm{~h}$, dark $8 \mathrm{~h}$ ). Drought treatment with $20 \%$ PEG was carried out under the conditions of $23^{\circ} \mathrm{C}$ room temperature, $12 \mathrm{~h}$ illumination, $12 \mathrm{~h}$ darkness and $62 \%$ humidity. After treatment, the roots, stems and leaves were quickly frozen in liquid nitrogen and stored at $-80^{\circ} \mathrm{C}$ for subsequent experiments.

\subsection{Total RNA extraction and first chain cDNA synthesis}

The total RNA of cassava was extracted by polysaccharide polyphenols plant total RNA extraction kit (catalog number: DP441). The integrity of the RNA was detected by agarose gel electrophoresis, and the quality of RNA was detected by ultramicro spectrophotometer. Taking 2 ug total RNA with better purity and concentration, then Fast Quant RT kit (catalog number: KR106) was used for reverse transcription synthesis of cDNA. 


\subsection{Cloning and quantitative real-time analysis of transcription factor}

Specific amplification primers were designed according to the transcription factor gene sequence of cassava MeTCP4 (Table 1). MeTCP4 gene was amplified using reverse transcription cDNA as template. The reaction system was $50 \mu \mathrm{L}$. The procedure for amplification of PCR was as follows: $94^{\circ} \mathrm{C} 3 \mathrm{~min}, 94^{\circ} \mathrm{C} 30 \mathrm{~s} 、 56^{\circ} \mathrm{C} 30 \mathrm{~s}$ 、 $72^{\circ} \mathrm{C} 1 \mathrm{~min} 20 \mathrm{~s}, 34$ cycles; $72^{\circ} \mathrm{C} 7 \mathrm{~min}$. The amplified products of PCR were detected by $1 \%$ agarose gel electrophoresis, the target fragments were then recovered and connected to the pCAMBIA1301 vector. Overnight culture resistance screening, single colony shaking bacteria were selected, and PCR liquid identification and positive liquid plasmid sequencing were carried out. The real-time fluorescence quantitative PCR primers and internal reference genes of cassava MeTCP4 transcription factors were designed according to cassava genome (Table 1). The relative expression of the genes was analyzed by SYBR Green fluorescent dye method. The instrument used was ABI7300 real-time fluorescence quantitative PCR. The reaction system of $20 \mu \mathrm{L}: 2 \times$ SYBR Premix Ex TaqII $10 \mu \mathrm{L}$, primer forward $0.8 \mu \mathrm{l}+$ primer reverse $0.8 \mu \mathrm{L}$ 、CDNA $1 \mu \mathrm{L}$ 、50xROX Reference Dye 0.4 $\mu \mathrm{L} 、 \mathrm{ddH}_{2} \mathrm{O} 7 \mu \mathrm{L}$; The reaction procedure was as follows: $95^{\circ} \mathrm{C} 30 \mathrm{~s}$ for 1 cycle, $95^{\circ} \mathrm{C} 5 \mathrm{~s}$ and $60^{\circ} \mathrm{C} 30 \mathrm{~s}$ for 40 cycles, repeating each sample three times, data processing using $2{ }^{-} \Delta \Delta \mathrm{Ct}$ method, then Microsoft Excel analyzed the output results and drew them.

Table 1 Cloning and expression of MeTCP4 transcription factor in cassava

\begin{tabular}{ll}
\hline Primer & Prime sequence $\left(5^{\prime}-3^{\prime}\right)$ \\
\hline MeTCP4-F & CGGGATCCTATGGGAGAGAGCCACCGCCAAG \\
MeTCP4-R & ACGCGTCGACTCAATGGCGAGAATCAGAGGA \\
MeTCP4 RT-F & AGTGCAGCCGATACTGGTAG \\
MeTCP4 RT-R & AATTCGTGCTGGAATGCGGA \\
Me Actin-F & TGGATTCTGGTGATGGTGTGAGT \\
Me Actin-R & CCGTTCAGCAGTGGTGGTGA \\
\hline
\end{tabular}

\subsection{Construction of gene plant expression vector}

The pCAMBIA1301 and rMeTCP4/ MeTCP4SRDX were double enzyme digested by BamH I and Sal I at $37^{\circ} \mathrm{C}$ for 2 hours respectively. Enzyme digestion product electrophoresis was used to detect the strips and gel was cut to recover the strips. DNA purification and recycling kit was used to recover the strips (catalogue number: 209). Solution 1 homologous recombinase was used to connect the carrier to the target product at $4^{\circ} \mathrm{C}$ for 12 hours. The plasmid was transferred into E. coli DH5 $\alpha$. The bacterial colonies were identified by PCR after overnight culture, then positive colonies were sequenced by Sangon Biotech.

\section{Authors' contributions}

LN was the executor of the experimental design and research of this study. He completed data analysis, experimental results analysis, and the writing of the first draft of the paper. LSX and PM were the conceivers and principal of the project, guiding experimental design, data analysis, paper writing and revision. All the authors read and agreed to the final text.

\section{Acknowledgments}

This study was supported by the National Natural Science Foundation of China (31561143012 and 31701484), the Program of Hainan Association for Science and Technology Plans to Youth R \& D Innovation (HAST201627), the Hainan Natural Science Foundation Innovation Team Project (YSH21605060336) and the Basic Research Business Fee (1630052017022) of the Institute of Tropical Biotechnology of the Chinese Academy of Tropical Agricultural Sciences.

\section{References}

Chen L.P., and He D.Y., 2010, Research advance on drought and salt resistant genes in transgenic plants, Jiyinzuxue Yu Yingyong Shengwuxue (Genomics and Applied Biology), 29(3): 542-549

Cock J.H., 1982, Cassava: a basic energy-source in the tropics, Science, 218(4574): 755-762 https://doi.org/10.1126/science.7134971

Cubas P., Lauter N., Doebley J., and Coen E., 1999, The TCP domain: a motif found in proteins regulating plant growth and development, Plant J., 18(2): 215-222

https://doi.org/10.1046/j.1365-313X.1999.00444.X 
Doebley J., Stec A., and Hubbard L., 1997, The evolution of apical dominance in maize, Nature, 386(6624): 485-488 https://doi.org/10.1038/386485a0

El-Sharkawy M.A., 1993, Drought-tolerant cassava for Africa, Asia, and Latin America: breeding projects work to stabilize productivity without increasing pressures on limited natural resources, Bioscience, 43(7): 441-451 https://doi.org/10.2307/1311903

El-Sharkawy M.A., 2004, Cassava biology and physiology, Plant Molecular Biology, 56: 481-501 https://doi.org/10.1007/s11103-005-2270-7

Hiratsu K., Matsui K., Koyama T., and Ohme-TakagiM., 2003, Dominant repression of target genes by chimeric repressors that include the EAR motif, a repression domain, in Arabidopsis, Plant J., 34: 733-739 https://doi.org/10.1046/j.1365-313X.2003.01759.X

Kosugi S., and Ohashi Y., 1997, PCF1 and PCF2 specifically bind to cis elements in the rice proliferating cell nuclear antigen gene, Plant Cell, 9(9): 1607-1619 https://doi.org/10.2307/3870447

Koyama T., Furutani M., Tasaka M., and Ohme-Takagi M., 2007, TCP transcription factors control the morphology of shoot lateral organs via negative regulation of the expression of boundary-specific genes in Arabidopsis, Plant Cell, 19: 473-484 https://doi.org/10.1105/tpc.106.044792

Luo D., Carpenter R., Vincent C., Copsey L., and Coen E., 1996, Origin of floral asymmetry in Antirrhinum, Nature, 383: 794-799 https://doi.org/10.1038/383794a0

Mahajan S., and Tuteja N., 2005, Cold, salinity and drought stresses: An overview, Archives of Biochemistry and Biophysics, 444(2): 136-158 https://doi.org/10.1016/j.abb.2005.10.018

Riechmann J.L., Heard J., Martin G., et al., 2000, Arabidopsis transcription factors: genome-wide comparative analysis among eukaryotes, Science, 290(5499): 2105-2110 https://doi.org/10.1126/science.290.5499.2105

Schommer C., Palatnik J.F., Aggarwal P., Chételat A., Cubas P., Farmer E.E., Nath U., and Weigel D., 2008, Control of jasmonate biosynthesis and senescence by miR319 targets, PLoS Biology, 6(9): e230 https://doi.org/10.1371/journal.pbio.0060230

Urano K., Kurihara Y., Seki M., and Shinozaki K., 2010, ‘Omics’ analyses of regulatory networks in plant abiotic stress responses, Curr. Opin. Plant Biol., 13(2): 132 https://doi.org/10.1016/j.pbi.2009.12.006

Wang S.T., Sun X.L., Hoshino Y., Yu Y., Jia B., Sun Z.W., Sun M.Z., Duan X.B., and Zhu Y.M., 2014, MicroRNA319 positively regulates cold tolerance by targeting OsPCF6 and OsTCP21 in rice, PLoS One, 9(3): e91357 https://doi.org/10.1371/journal.pone.0091357

Zhang F., Li R.M., Yuan S., Geng M.T., Yao Y., Liu J., Duan R.J., Fu S.P., Hu X.W., and Guo J.C., 2017, Jiyinzuxue Yu Yingyong Shengwuxue (Genomics and Applied Biology), 36(4): 1581-1587

Zhou M., Li D.Y., Li Z.G., Hu Q., Yang C.H., Zhu L.H., and Luo H., 2013, Constitutive expression of a miR319 gene alters plant development and enhances salt and drought tolerance in transgenic creeping bentgrass, Plant Physiol., 161: 1375-1391 https://doi.org/10.1104/pp.112.208702 